\title{
PROFICIENT PRESENTATIONS AND DIRECT PRODUCTS OF FINITE GROUPS
}

\author{
K.W. Gruenberg and L.G. Kovács \\ To Bernhard Neumann on the occasion of his 90th birthday, \\ with gratitude and affection
}

Let $G$ be a finite group, $F$ a free group of finite rank, $R$ the kernel of a homomorphism $\varphi$ of $F$ onto $G$, and let $[R, F],[R, R]$ denote mutual commutator subgroups. Conjugation in $F$ yields a $G$-module structure on $R /[R, R]$; let $d_{G}(R /[R, R])$ be the number of elements required to generate this module. Define $d(R /[R, F])$ similarly. By an earlier result of the first author, for a fixed $G$, the difference $d_{G}(R /[R, R])-d(R /[R, F])$ is independent of the choice of $F$ and $\varphi$; here it is called the proficiency gap of $G$. If this gap is 0 , then $G$ is said to be proficient. It has been more usual to consider $d_{F}(R)$, the number of elements required to generate $R$ as normal subgroup of $F$ : the group $G$ has been called efficient if $F$ and $\varphi$ can be chosen so that $d_{F}(R)=d_{G}(R /[R, F])$. An efficient group is necessarily proficient; but (though usually expressed in different terms) the converse has been an open question for some time.

The first part of the paper discusses similar issues in the category of profinite groups and continuous homomorphisms. One of the conclusions is that a finite group is proficient as discrete group if and only if it is efficient as profinite group.

Returning to the discrete setting, the second part explores the proficiency of a direct product in terms of conditions on the direct factors.

\section{INTRODUCTION}

There are three natural integers associated with every finite free presentation of a finitely presentable group. If $F / R \approx G$ is such a presentation, then these numbers are, in order of decreasing size, $d_{F}(R), d_{G}(R /[R, R]), d(R /[R, F])$. (Our notation is standard: if $P$ is a group of operators on a group $X$, then $d_{P}(X)$ denotes the minimum number of elements of $X$ needed to generate $X$ as $P$-group.) It is convenient (and usual) to involve $d(F)$ and we define the defect of the presentation to be $\operatorname{def} F / R=$ $d_{F}(R)-d(F) ;$ the abelianised defect to be adef $F / R=d_{G}(R /[R, R])-d(F)$; and the

Received 24th February, 1999

The authors are indebted to M.F. Newman for his examples reported in the last section of this paper and for many illuminating discussions.

Copyright Clearance Centre, Inc. Serial-fee code: 0004-9727/99 \$A2.00+0.00. 
centralised defect to be cdef $F / R=d(R /[R, F])-d(F)$. This last is an invariant of $G$ (meaning it is independent of the choice of $F / R$ ) because

$$
\text { cdef } F / R=d\left(H_{2}(G, \mathbb{Z})\right)-\operatorname{dim}_{\mathbb{Q}} \mathbb{Q} \otimes(G /[G, G]) .
$$

We may therefore write it as cdef $G$.

Abelianised defects can vary with the choice of free presentation: recall Dunwoody's famous result [6] that $\left\langle x, y ! x^{2}=y^{3}\right\rangle$ has a non-obvious free presentation with abelianised defect 0 . However, if $G$ is finite, then adef $F / R$ is independent of $F / R$ [7], giving the invariant adef $G$.

We remark that if $G$ is any finitely presentable group, $\{$ adef $F / R \mid F / R \simeq G\}$ is bounded below, whence its lower bound may be taken as defining adef $G$. Then $\operatorname{def} G$ will denote the lower bound of all $\operatorname{def} F / R$. But our interest in this note is exclusively with finite groups. Note that here $\operatorname{cdef} G=d\left(H_{2}(G, \mathbb{Z})\right)$, whence $\operatorname{def} G \geq 0$.

The existence of a finite (or even finitely presentable) group $G$ having a free presentation $F / R \simeq G$ with $\operatorname{def} F / R>$ adef $F / R$ remains open (this is the relation gap problem). There is even the tantalising possibility that such a free presentation exists and yet $\operatorname{def} G=\operatorname{adef} G$.

The difference adef $F / R-\operatorname{cdef} F / R$ is much better understood. We shall call this the proficiency gap of the finite group $G$; and when this is 0 , we say $G$ is proficient.

To explain this terminology we point out that our finite group $G$ lives not only in the universe of discrete groups but also in that of profinite groups. Every finite free presentation $F / R \simeq G$ in the category of profinite groups yields invariants of the same type as those discussed above. We shall write these as def, âdef, ĉdef respectively, and shall prove that always

(1) $\operatorname{def} F / R=$ âdef $F / R$; and

(2) âdef $F / R=\operatorname{adef} G$ (whence is independent of $F / R$ ). Moreover,

(3) $\hat{c} \operatorname{def} F / R=\operatorname{cdef} G$.

Thus there is no profinite relation gap problem and proficiency has the same meaning in both categories. A discrete $G$ is called efficient if $\operatorname{def} G=\operatorname{cdef} G$. Adopting the same term in the profinite category, we have that the finite group $G$ is efficient as profinite group if, and only if, $G$ is proficient.

After these preliminaries (proofs of (1), (2) and (3) are in Section 1), we turn to our main interest here: how proficiency behaves under direct products. Recall that a group $G$ is called superperfect if $G$ is perfect (that is, $H_{1}(G, \mathbb{Z})=0$ ) and $H_{2}(G, \mathbb{Z})=0$. Also, $G^{(n)}$ shall mean the direct product $G \times \cdots \times G$ with $n$ factors. We shall prove (all groups are finite) that

(4) for any $G$ and any non-superperfect $H, E=G \times H^{(n)}$ is proficient for all sufficiently large $n(2.6)$; 
(5) if $E=G \times H$ is perfect and $d_{E}(\Delta E)=2$ (where $\Delta E$ denotes the augmentation ideal of $\mathbb{Z} E$ ), then $G, H$ proficient implies $E$ proficient (2.8);

(6) if $E=G \times H$ is superperfect, then $E$ proficient implies $G, H$ proficient (2.9);

(7) if $G$ is superperfect and $n>1$, then $G^{(n)}$ is proficient if, and only if, $G \times G$ is proficient if, and only if, $G$ is proficient and $d_{G}(\Delta G)=2(2.10)$.

A special case of (4) is quoted in Harlander's paper [10]. Related arguments occur also in Campbell, Robertson, Williams [3]. Results (4)-(7) are proved in Section 2. The last two sections deal with some questions raised by our results and give examples to illustrate them.

\section{Profinite groups}

When dealing with profinite groups, terms such as subgroup, normal subgroup, commutator subgroup, et cetera, are always to be understood in the profinite sense.

(1.1) If $H$ is a normal subgroup of the finitely generated profinite group $G$ and $a_{1}, \ldots, a_{n}$ are elements in $H$ whose normal closure $A$ in $G$ satisfies $A H^{\prime}=H$ (where $H^{\prime}$ is the commutator subgroup of $H$ ), then there exists $c$ in $H^{\prime}$ such that $H$ is the normal closure of $a_{1} c, a_{2}, \ldots, a_{n}$.

ProOf: Let us write the normal closure of a subset $X$ of $G$ as $\langle G\langle X\rangle$. Thus $A=\left\langle G\left\langle a_{1}, \ldots, a_{n}\right\rangle\right.$ and we are given $A H^{\prime}=H$, whence $H / A$ is perfect.

Since $G$ is finitely generated, $G=\varliminf G / N_{i}$, where the $N_{i}$ form a descending chain of open normal subgroups with $i \in \mathbb{Z}_{\geq 0}$ and $N_{0}=G$. Then $H=\lim H / M_{i}$, where $M_{i}=N_{i} \cap H$ and $M_{0}=H$. Find the smallest integer $r \geq 1$ so that $A M_{r}<H$ : if no such $r$ exists, then $A M_{i}=H$ for all $i \geq 1$, whence $A=H$ and we are done. Thus $A M_{r-1}=H$ and we use the following result (whose proof we postpone):

(1.2) With $H, A$ as above, let $K$ be a $G$-invariant open subgroup of $H$ satisfying $A K=H$. If $M$ is any $G$-invariant open subgroup of $H$ contained in $K$, then there exists $x$ in $K^{\prime}$ such that $\left\langle G\left\langle a_{1} x, a_{2}, \ldots, a_{n}\right\rangle M=H\right.$.

Returning to $A M_{r-1}=H$, we can find $x \in M_{r-1}^{\prime} \leq M_{0}^{\prime}=H^{\prime}$ so that $A_{r} M_{r}=H$, where $A_{r}=\left\langle G\left\langle a_{1} x, a_{2}, \ldots, a_{n}\right\rangle\right.$. If $A_{r} \neq H$, we take $s$ to be the smallest integer exceeding $r$ so that $A_{\mathrm{r}} M_{s}<H$. Then $A_{r} M_{s-1}=H$ and, again by (1.2), there is an element $y$ in $M_{s-1}^{\prime}$ so that $A_{s} M_{s}=H$, where $A_{s}=\left\langle G\left\langle a_{1} x y, a_{2}, \ldots, a_{n}\right\rangle\right.$.

Changing notation, we obtain a sequence of $G$-invariant open subgroups of $H$,

$$
H=L_{0}>L_{1}>L_{2}>\cdots
$$

(in the above notation, $L_{0}=M_{0}, L_{1}=M_{r}, L_{2}=M_{s}$ ) and elements $c_{i} \in L_{i}^{\prime}$ such that $\left\langle G\left\langle a_{1} c_{1} \cdots c_{k}, a_{2}, \ldots, a_{n}\right\rangle L_{k}=H\right.$. Now $\left(c_{1}, c_{1} c_{2}, c_{1} c_{2} c_{3}, \ldots\right)$ is a Cauchy 
sequence in $H$ (since $\bigcap L_{i}=1$ ), whence it converges, say to $c$. Thus $c \in H^{\prime}$ and $\left\langle G\left\langle a_{1} c, a_{2}, \ldots, a_{n}\right\rangle L_{k}=H\right.$ for all $k$, which establishes (1.1).

PROOF OF (1.2): This is a result about a finite normal subgroup of a profinite group, because we may work modulo $M$. Thus $H$ is finite and we take a sequence of $G$-invariant subgroups from $K$ to 1 , of maximal length: say

$$
K=M_{0}>M_{1}>\cdots>M_{r}=1
$$

where $M_{i} / M_{i+1}$ is minimal normal in $G / M_{i+1}$. We prove our result by induction on $r$. So we may assume $K$ is minimal normal in $G$. Then $A \cap K=1$ as otherwise $K \leq A$, whence $A=H$. Now $H=A \times K$ and $K \simeq H / A$ is perfect. We may choose $x$ in $K^{\prime}=K$ so that $x$ does not belong to any proper normal subgroup of $K$. Then the normal closure of $\left[a_{1} x, K\right]=[x, K]$ is equal to $K$, whence $\left\langle G\left\langle a_{1} x, a_{2}, \ldots, a_{n}\right\rangle\right.$ contains $K$ and so equals $H$.

An immediate consequence of (1.1) is

(1.3) Let $F / R \simeq G$ be a finite free presentation of the finitely presentable profinite group $G$. Then $d_{F}(R)=d_{G}\left(R / R^{\prime}\right)$.

Of course, $d_{F}(R)$ denotes the minimum number of generators of $R$ as profinite $F$-group; and similarly for the topological $G$-module $R / R^{\prime}$. Thus in the notation of our introduction, (1.3) tells us that

(1.4) $\hat{\operatorname{def}} F / R=$ âdef $F / R$.

Now assume $G$ is a finite group and consider $F / R \simeq G$, with $F$ the free profinite group on $x_{1}, \ldots, x_{d}$. If $F_{0}$ is the discrete subgroup generated by $x_{1}, \ldots, x_{d}$, then $F_{0}$ is the free (discrete) group on these elements. Let $R_{0}=F_{0} \cap R$. Then $R$ is the closure

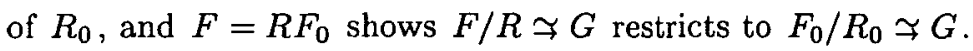

It is an elementary fact (for example, $[14,5.4 .4]$ ) that if $Y$ is a free generating set of $R_{0}$ constructed from $x_{1}, \ldots, x_{d}$ in the standard way by means of a Schreier transversal to $R_{0}$ in $F_{0}$, then $R$ is the free profinite group on $Y$. Hence $d(R)=d\left(R_{0}\right)$.

(1.5) Given $F / R \Im G$ and $F_{0}, R_{0}$ as above, then

$$
\text { âdef } F / R=\operatorname{adef} F_{0} / R_{0} \text {. }
$$

ProOF: We have a natural profinite surjection $\varphi: R \rightarrow R / R^{\prime} R^{p}$ for any prime $p$, since $R^{\prime} R^{p}$ is open in $R$. This also implies $R_{0}\left(R^{\prime} R^{p}\right)=R$, which shows $\varphi$ restricts to a surjection $R_{0} / R_{0}^{\prime} R_{0}^{p} \rightarrow R / R^{\prime} R^{p}$. But

$$
\operatorname{dim}_{\mathbb{F}_{p}} R / R^{\prime} R^{p}=d(R)=d\left(R_{0}\right)=\operatorname{dim}_{F_{p}} R_{0} / R_{0}^{\prime} R_{0}^{p}
$$


and so we have the commutative square

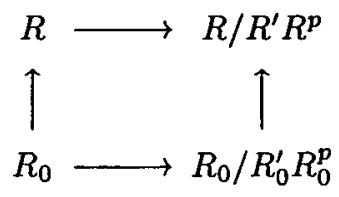

in which the horizontal maps are surjective, the left vertical map is inclusion, and the right vertical map is an isomorphism of $\mathbb{F}_{p} G$-modules. Our prime $p$ can be chosen so that $d_{G}\left(R_{0} / R_{0}^{\prime}\right)=d_{G}\left(R_{0} / R_{0}^{\prime} R_{0}^{p}\right.$ ) (see [7] or [8], Sections $7.3,7.4$ for a less terse account of these matters). Then

$$
\begin{array}{rlrl}
d_{G}\left(R / R^{\prime}\right) & \leq d_{G}\left(R_{0} / R_{0}^{\prime}\right), & & \text { because } R_{0} R^{\prime} / R^{\prime} \text { is dense in } R / R^{\prime} \\
& =d_{G}\left(R_{0} / R_{0}^{\prime} R^{p}\right) & \\
& =d_{G}\left(R / R^{\prime} R^{p}\right), & & \text { by the commutative square above } \\
& \leq d\left(R / R^{\prime}\right), & & \text { obviously. }
\end{array}
$$

Hence we have equality throughout and (1.5) is proved.

In view of the fact that adef $F_{0} / R_{0}$ is constant, (1.5) yields

(1.6) âdef $F / R$ is independent of $F / R$ and its value equals adef $G$.

Note that we established (1.4) for any finitely presentable profinite group $G$, but (1.6) only for finite $G$. We leave open the question whether (1.6) remains true for non-finite profinite groups.

It remains to prove

(1.7) In the notation of (1.5), ĉdef $F / R=$ cdef $F_{0} / R_{0}$.

PROOF: The argument is essentially the same as for (1.5) but is entirely elementary.

Since $R_{0}$ is dense in $R$ and $F_{0}$ is dense in $F$, therefore $\left[R_{0}, F_{0}\right]$ is dense in $[R, F]$. So the inclusion $R_{0} \rightarrow R$ induces a surjection $R_{0} /\left[R_{0}, F_{0}\right] R_{0}^{p} \rightarrow R /[R, F] R^{p}$ for every prime $p$. This is an isomorphism because $R_{0}$ and $R$ are free on the same set $Y$ and the image of $Y$ is a basis of $R_{0} /\left[R_{0}, F_{0}\right] R_{0}^{p}$ and also of $R /[R, F] R^{p}$. Thus

$$
d\left(R /[R, F] R^{p}\right)=d\left(R_{0} /\left[R_{0}, F_{0}\right] R_{0}^{p}\right)
$$

Choose $p$ so that $d\left(R_{0} /\left[R_{0}, F_{0}\right]\right)=d\left(R_{0} /\left[R_{0}, F_{0}\right] R_{0}^{p}\right)$. Then

$$
d(R /[R, F]) \leq d\left(R_{0} /\left[R_{0}, F_{0}\right]\right)=d\left(R /[R, F] R^{p}\right) \leq d(R /[R, F])
$$

and we are done. 


\section{DIRECT PRODUCTS}

Our basic tools are the first two partial Euler characteristics $\nu_{1}(G)$ and $\nu_{2}(G)$ of the finite group $G$. Let us recall some facts from [9].

By $[9,(1)$ and $(2)]$, we have

(2.1) $\nu_{1}(G)=d_{G}(\Delta G)-1$ and $\nu_{2}(G)=\operatorname{adef} G+1$.

Hence

(2.2) $G$ is proficient if, and only if, $\nu_{2}(G)=1+d\left(H_{2}(G, \mathbb{Z})\right)$.

To calculate $\nu_{2}(G)$ one proceeds as follows. For each prime $p$ dividing $|G|$ (the other primes are irrelevant) choose a finite splitting field $K(p)$ for $G$, and all its subgroups, of characteristic $p$; for each irreducible $K(p) G$-module $M$ set

$$
\nu_{2}(G, M)=\left\lceil\frac{1}{\operatorname{dim} M}\left(\operatorname{dim} H^{2}(G, M)-\operatorname{dim} H^{1}(G, M)+\operatorname{dim} H^{0}(G, M)\right)\right\rceil
$$

(where $\lceil a\rceil$ means the smallest integer $\geq a$ ), and

$$
\nu_{2}(K(p) G)=\max \left(\nu_{2}(G, M) \mid \text { all } M\right) .
$$

Then $\nu_{2}(G)=\max \left(\nu_{2}(K(p) G) \mid\right.$ all $\left.p\right)$. (See $[9,(7)]$.)

It is clear (or see $[9,(6)]$ ) that $\nu_{2}(G, K(p))=\nu_{2}\left(G, \mathbb{F}_{p}\right)$ and (see $\left.[9,(13)]\right)$

$$
\nu_{2}\left(G, \mathbb{F}_{p}\right)=1+\operatorname{dim}\left(H_{2}(G, \mathbb{Z}) / p H_{2}(G, \mathbb{Z})\right) \text {. }
$$

Let $E=G_{1} \times \cdots \times G_{n}$ and $K$ be a finite splitting field for $E$. Every irreducible $K E$-module $A$ has the form $A=A_{1} \sharp \cdots \sharp A_{n}$ (outer tensor product), where each $A_{i}$ is an irreducible $K G_{i}$-module, and every such product is $K E$-irreducible (for example, $[5,10.33])$.

For every $q \geq 1$,

$$
H^{q}(E, A)=\bigoplus_{\sum q_{i}=q}\left(\bigotimes H^{q_{i}}\left(G_{i}, A_{i}\right)\right)
$$

(for example, [9], the argument on p.271 leading to (8)).

Suppose now that in $A=A_{1} \sharp \cdots \sharp A_{n}$, the modules $A_{1}, \ldots, A_{m}$ are non-trivial and $A_{m+1}=\cdots=A_{n}=K(m \geq 0)$. Thus $H^{0}\left(G_{i}, A_{i}\right)=0$ for $i \leq m$ and so (2.4) gives

$$
H^{1}(E, A)= \begin{cases}0 & \text { if } m \geq 2 \\ H^{1}\left(G_{1}, A_{1}\right) & \text { if } m=1 \\ \bigoplus_{i=1}^{n} H^{1}\left(G_{i}, K\right) & \text { if } m=0\end{cases}
$$


and

$$
H^{2}(E, A)= \begin{cases}0 & \text { if } m \geq 3, \\ H^{1}\left(G_{1}, A_{1}\right) \otimes H^{1}\left(G_{2}, A_{2}\right) & \text { if } m=2, \\ \bigoplus_{i=2}^{n}\left(H^{1}\left(G_{1}, A_{1}\right) \otimes H^{1}\left(G_{i}, K\right)\right) \oplus H^{2}\left(G_{1}, A_{1}\right) & \text { if } m=1 \\ \bigoplus_{i<j}\left(H^{1}\left(G_{i}, K\right) \otimes H^{1}\left(G_{j}, K\right)\right) \oplus\left(\bigoplus_{i} H^{2}\left(G_{i}, K\right)\right) & \text { if } m=0\end{cases}
$$

Writing $a_{i}=\operatorname{dim} A_{i}, h^{k}\left(A_{i}\right)=\operatorname{dim} H^{k}\left(G_{i}, A_{i}\right), h^{k}\left(G_{i}\right)=\operatorname{dim} H^{k}\left(G_{i}, K\right)$ we now have

(2.5) If $A=A_{1} \sharp \cdots \sharp A_{m} \sharp K \sharp \cdots \sharp K$ with $A_{i} \neq K$, then

$$
\nu_{2}(E, A)= \begin{cases}0 & \text { if } m \geq 3, \\ \left\lceil\frac{h^{1}\left(A_{1}\right) h^{1}\left(A_{2}\right)}{a_{1} a_{2}}\right]=\nu_{2}\left(G_{1} \times G_{2}, A_{1} \sharp A_{2}\right) & \text { if } m=2, \\ \left\lceil\frac{1}{a_{1}}\left(h^{1}\left(A_{1}\right)\left(\sum_{i \geq 2} h^{1}\left(G_{i}\right)\right)+h^{2}\left(A_{1}\right)-h^{1}\left(A_{1}\right)\right)\right] & \text { if } m=1, \\ \sum_{i<j} h^{1}\left(G_{i}\right) h^{1}\left(G_{j}\right)+\sum_{i}\left(h^{2}\left(G_{i}\right)-h^{1}\left(G_{i}\right)\right)+1 & \text { if } m=0 .\end{cases}
$$

All our results on direct products flow from (2.5).

Suppose first that $E=G \times H^{(r)}$, where $H$ is assumed not superperfect. So (2.5) with $n=r+1, G_{1}=G$ and $G_{i}=H$ for $i \geq 2$, shows $\nu_{2}(K(p) E)$ is the maximum of all

(i)

$$
\begin{gathered}
\nu_{2}\left(G \times H, A_{1} \sharp A_{2}\right), \quad \nu_{2}\left(H \times H, A_{2} \sharp A_{3}\right), \\
\left\lceil\frac{1}{a_{1}}\left(r h^{1}\left(A_{1}\right) h^{1}(H)+h^{2}\left(A_{1}\right)-h^{1}\left(A_{1}\right)\right)\right], \\
\left\lceil\frac{1}{a_{2}}\left((r-1) h^{1}\left(A_{2}\right) h^{1}(H)+h^{1}(G) h^{1}\left(A_{2}\right)+h^{2}\left(A_{2}\right)-h^{1}\left(A_{2}\right)\right)\right\rceil,
\end{gathered}
$$

(iii) $r h^{1}(G) h^{1}(H)+\frac{r(r-1)}{2} h^{1}(H)^{2}+h^{2}(G)-h^{1}(G)+r\left(h^{2}(H)-h^{1}(H)\right)+1$.

Thus $\nu_{2}(E)$ is the maximum of these expressions as $p$ is also allowed to vary over the prime divisors of $|G||H|$. As functions of $r$, these expressions are (finitely many) polynomials of degree at most 2 , so one of them will dominate the others for all sufficiently large $r$. If $h^{1}(H) \neq 0$ for at least one choice of $p$, we have at least one polynomial with positive leading coefficient and degree precisely 2 , and the dominant one will have to be one of these. Since all these are of type (iii), so is the dominant one. If $h^{1}(H)=0$ 
for all $p$, then $H_{1}(H, \mathbb{Z})=0$, so $H_{2}(H, \mathbb{Z}) \neq 0$, and then $(2.3)$ shows that $h^{2}(H) \neq 0$ for at least one choice of $p$. Now there is no quadratic, but there is at least one linear polynomial with positive leading coefficient, and all these are of type (iii), so in this case also the dominant polynomial comes from (iii). Say, $p_{0}$ is one of the (possibly several) characteristics where (iii) gives this dominant polynomial. Then, for $r$ sufficiently large,

$$
\nu_{2}\left(E, K\left(p_{0}\right)\right)=\nu_{2}\left(K\left(p_{0}\right) E\right) \geq \nu_{2}(K(p) E) \text { for all } p
$$

and so

$$
\nu_{2}(E)=\max _{p} \nu_{2}\left(E, \mathbb{F}_{p}\right)=1+\max _{p} \operatorname{dim}\left(H_{2}(E, \mathbb{Z}) / p H_{2}(E, \mathbb{Z})\right)=1+d\left(H_{2}(E, \mathbb{Z})\right) .
$$

(2.6) If $H$ is not superperfect, then $G \times H^{(r)}$ is proficient for all sufficiently large $r$.

Return to the general case $E=G_{1} \times \cdots \times G_{n}$ but now assume $E$ is perfect. Here (2.5) with $m=1$ is

$$
\left\lceil\frac{1}{a_{1}}\left(h^{2}\left(A_{1}\right)-h^{1}\left(A_{1}\right)\right)\right\rceil=\nu_{2}\left(G_{1}, A_{1}\right)=\nu_{2}\left(G_{1} \times G_{2}, A_{1} \sharp K\right) ;
$$

and $H^{2}(E, K)=\bigoplus_{i} H^{2}\left(G_{i}, K\right)$ (by $(2.4)$ ), whence $\nu_{2}\left(G_{1} \times G_{2}, K\right) \leq \nu_{2}(E, K)$. This gives the first part of

(2.7) Assume $E=G_{1} \times \cdots \times G_{n}$ is perfect.

(i) $\nu_{2}(E)=\max \left(\nu_{2}\left(G_{i} \times G_{j}\right)\right.$ for all $\left.i<j ; 1+d\left(H_{2}(E, \mathbb{Z})\right)\right)$.

(ii) If $\nu_{1}\left(K(p) G_{i}\right) \nu_{1}\left(K(p) G_{j}\right) \leq 1+d\left(H_{2}(E, \mathbb{Z})\right.$ ) for all $p$ and all $i<j$, then $\nu_{2}(E)=\max \left(\nu_{2}\left(G_{i}\right)\right.$ for all $\left.i ; 1+d\left(H_{2}(E, \mathbb{Z})\right)\right)$.

PROOF of (ii): Since $G_{i}$ is perfect, $\nu_{1}\left(G_{i}\right)$ is the maximum of $\left\lceil h^{1}\left(A_{i}\right) / a_{i}\right\rceil$ for all $A_{i} \neq K(p)$ and all $p$. Thus our hypothesis and (2.5) show that if $m=2$, then $\nu_{2}(E, A) \leq 1+d\left(H_{2}(E, \mathbb{Z})\right)$. We already know that $\nu_{2}(E, A)=\nu_{2}\left(G_{1}, A_{1}\right)$ if $m=1$. Also, $\nu_{2}\left(G_{i}, K(p)\right) \leq \nu_{2}(E, K(p)) \leq 1+d\left(H_{2}(E, \mathbb{Z})\right)$ for all $p$, and $\nu_{2}(E, K(p))=$ $1+d\left(H_{2}(E, \mathbb{Z})\right)$ for at least one $p$. As $\nu_{2}(E)$ is the maximum of the $\nu_{2}(E, A)$ over all $p$ and all simple $K(p) E$-modules $A$, our result follows.

(2.7)(i) shows, using also $H_{2}(E, \mathbb{Z})=\bigoplus_{i} H_{2}\left(G_{i}, \mathbb{Z}\right)$, that if $E$ is perfect, then the proficiency of all $G_{i} \times G_{j}$ implies the proficiency of $E$. We shall see that the converse is true when $E$ is superperfect (2.9); but in the non-superperfect case nothing obvious seems possible: take any perfect direct product $G_{1} \times G_{2}$ and any perfect, but not superperfect $H$; then $G_{1} \times G_{2} \times H^{(r)}$ is proficient for sufficiently large $r$ (by (2.6)).

Along the same lines, (2.7)(ii) shows $E$ is proficient if all $G_{i}$ are proficient provided the hypotheses of (ii) hold. Recall from (2.1) that these hypotheses are size restrictions on $d_{G_{i}}\left(\Delta G_{i}\right)$. Since each $G_{i}$ is perfect, $d_{G_{i}}\left(\Delta G_{i}\right) \geq 2$. If $d_{G_{i}}\left(\Delta G_{i}\right)=2$ for all $i$, there is no restriction and we have 
(2.8) Assume $E=G_{1} \times \cdots \times G_{n}$ is perfect and that each $d_{G_{i}}\left(\Delta G_{i}\right)=2$. Then $G_{i}$ proficient for all $i$ implies $E$ is proficient.

The converse question is more subtle and we shall deal only with the superperfect case.

(2.9) If $E=G_{1} \times \cdots \times G_{n}$ is superperfect, then $E$ proficient implies that each $G_{i}$ is proficient.

This is easy: $\nu_{2}(E)=1$ gives $\nu_{2}\left(G_{i}, A_{i}\right) \leq 1$ by $(2.5)$ with $m=1$ and so $\nu_{2}\left(G_{i}\right)=1$.

(2.8) and (2.9) combine with the old result [4, Theorem 2] that

$$
d_{E}(\Delta E)=\max \left(d_{G_{i}}\left(\Delta G_{i}\right), \text { all } i\right)
$$

to show that a direct product is superperfect, proficient and has 2 -generator augmentation ideal if, and only if, each direct factor has the same properties.

Does the proficiency of a superperfect direct product already impose restrictions on the number of generators of its augmentation ideal? We return to this question in the next section. The only general result we have in this direction is

(2.10) If $G$ is superperfect then $G \times G$ is proficient if, and only if, $G$ is proficient and $d_{G}(\Delta G)=2$.

To complete the proof of (2.10) we only need to show that the proficiency of $G \times G$ implies $d_{G}(\Delta G)=2$, that is, that $\nu_{2}(G \times G)=1$ implies that $\nu_{1}(G)=1$. If $A$ is a non-trivial $K G$-module ( $K$ being, as usual, a suitable splitting field) then $\nu_{2}(G \times G, A \sharp A)=\left\lceil\left(h^{1}(A) / a\right)^{2}\right\rceil \leq 1$ shows $h^{1}(A) / a \leq 1$, which is what we need.

\section{MORE ON SUPERPERFECT GROUPS}

We recall that superperfect groups with arbitrarily large proficiency gap were constructed in [12] and that by (2.9) every direct product of superperfect groups is nonproficient provided one factor is non-proficient.

Suppose, on the other hand, that $E=G \times H$ is superperfect and $G, H$ are proficient. We know from (2.7)(ii) that the condition $\nu_{1}(K(p) G) \nu_{1}(K(p) H) \leq 1$ for all $p$, is sufficient to make $E$ proficient. If $G$ and $H$ could be constructed to be like this, but in such a way that, as $p$ varies, one of $\nu_{1}(K(p) G)$ and $\nu_{1}(K(p) H)$ is very small whenever the other is large, then one could hope to obtain $G$ and $H$ with neither having a 2-generator augmentation ideal (see (2.1)).

A related question is whether it is sufficient for the proficiency of $E$ to assume that at least one of $G, H$ has 2-generator augmentation ideal. In this direction we shall show (3.1) that to each superperfect, proficient group $G$, irrespective of the value 
of $d_{G}(\Delta G)$, there is a superperfect, proficient group $H$ such that $G \times H$ is proficient. Let $H_{r}=S L(2, r)$, where $r$ is a prime such that $r \equiv 3(\bmod 4)$ and $r>3$. Since $H_{r}$ has a 2-generator, 2-relator presentation [2], $H_{r}$ is superperfect, proficient, and $d_{H_{r}}\left(\Delta H_{r}\right)=2$.

(3.1) If $G$ is any superperfect, proficient group, then $G \times H_{r}$ is proficient for all sufficiently large $r$.

To prove this we need

(3.2) If $A$ is an absolutely simple $H_{r}$-module whose characteristic is different from $r$, then $h^{1}(A) / a \leq 10 /(r-1)$.

(In fact, characteristic $r$ need not be excluded and much better bounds must be well known, but even this weak result will be good enough here.)

Proof: We can assume that $A$ is non-trivial (else $h^{1}(A)=0$, because $H_{r}$ is perfect). Consider any element $h$ of order $r$ in $H_{r}$. This element does not lie in any proper normal subgroup, so must act nontrivially on $A$. Since the characteristic of $A$ is not $r, h$ has an eigenvalue $\lambda$ on $A$ which is a primitive $r$ th root of 1 . As $h$ is conjugate in $H_{r}$ to all its powers to square exponents, it follows that all powers of $\lambda$ to square exponents are eigenvalues of $h$ on $A$. This exhibits $(r-1) / 2$ distinct eigenvalues, whence $a \geq(r-1) / 2$. It remains to prove that $h^{1}(A) \leq 5$.

All odd order Sylow subgroups of $H_{r}$ are cyclic, therefore in odd characteristic all semisimple sections of the projective indecomposable modules are multiplicity-free (see Proposition 21.6 in Alperin [1]). Thus it follows from [8, Lemma 2.11] that if the characteristic of $A$ is odd then $h^{1}(A) \leq 1$. Now consider characteristic 2. The $H_{r}$-module induced from the 1 -dimensional trivial module of an odd order subgroup of index $2(r+1)$ is projective, and the projective cover of the 1-dimensional trivial $H_{r}$-module is a direct summand of that, so this projective cover has dimension at most $2(r+1)$. So if $A$ occurs $s$ times as a composition factor of this projective module, $a s+2 \leq 2(r+1)$ and therefore $((r-1) s) / 2 \leq 2 r$, whence $s \leq 5$, because $r \geq 5$. Again by $[8,2.11]$ we now have $h^{1}(A) \leq 5$. The proof of $(3.2)$ is complete.

Proof OF (3.1): Choose $r \equiv 3(\bmod 4)$ and such that $r \geq \max \left\{|G|, 10 d_{G}(\Delta G)\right\}$. By $(3.2), \nu_{1}\left(K(p) H_{r}\right) \leq 10 /(r-1)$ provided $p \neq r$. Moreover, $\nu_{1}(K(p) G) \leq$ $(r-10) / 10$ for all $p$, while $\nu_{1}(K(r) G)=0$. Hence $\nu_{1}(K(p) G) \nu_{1}\left(K(p) H_{r}\right) \leq 1$, and $(3.1)$ is proved.

\section{SOME EXAMPLES}

The most intriguing problem concerning presentations of finite groups remains whether there exist finite groups with positive relation gap. Such a group cannot be efficient; it may or may not be proficient; the only connection with proficiency is that 
a proficient group which is not efficient must have positive relation gap. Thus one fragment of the problem is: must every proficient group be efficient? We expect that the answer may be negative, and that relevant examples need not be too complicated. This would be in line with the experience that Swan's examples (see below) were completely transparent once they were recognised.

It was shown by Kenne [11] that the smallest non-efficient group is the semidirect product $\mathcal{G}$ of the rank 2 free group of exponent 3 with a group of order 2 , the nontrivial element of the latter inverting both free generators of the former. Since this group is not perfect, by (2.6) all sufficiently large direct powers of it are proficient. It is straightforward to calculate that the proficiency gaps of $\mathcal{G}, \mathcal{G}^{(2)}$ and $\mathcal{G}^{(3)}$ are 1,2 and $\mathbf{0}$, respectively, so the first proficient direct power is $\mathcal{G}^{(\mathbf{3})}$. Wotherspoon [15] had already drawn attention to the question whether this group is efficient, but at the time of writing this seems to be still open.

We also know from (2.6) that $\mathcal{G} \times C_{2}^{(n)}$ (as usual, $C_{k}$ stands for the cyclic group of order $k$ ) is proficient for all large $n$. In fact, Wotherspoon [15] proved that this group is efficient whenever $n>0$. There are no surprises among the $\mathcal{G} \times C_{p}^{(n)}$ for odd $p$ either.

Other small non-efficient groups come from the infinite sequence of groups $G_{k}$ of order $7^{k} 3$ constructed by Swan in [13]. These are famous as the first examples of groups with $H_{2}\left(G_{k}, \mathbb{Z}\right)=0$ but adef $G_{k} \rightarrow \infty$. In fact, adef $G_{1}=\operatorname{adef} G_{2}=0$ while adef $G_{3}=2$, so $G_{3}$ is the smallest non-proficient group among them. Before turning to that, let us note that while $G_{2}$ is proficient, its direct square is not, but then all higher direct powers are. It would be interesting therefore to decide whether these higher direct powers are efficient. Let us ask specifically: is $G_{2}^{(3)}$ efficient?

Our (2.6) shows that $G_{3} \times C_{3}^{(n)}$ is proficient for all sufficiently large $n$. By Corollary 5.4 of Harlander [10] (see also the note added in proof there), if $n$ is large enough then $G_{3} \times C_{3}^{(n)}$ is not only proficient but even efficient. If 'large enough' meant different things in these two contexts, we would have a finite group with positive relation gap. It is not hard to calculate that the proficiency gaps of $G_{3}, G_{3} \times C_{3}$ and $G_{3} \times C_{3}^{(2)}$ are 2, 1 and 0 , respectively, so the first question is whether $G_{3} \times C_{3}^{(2)}$ is efficient. As $H_{2}\left(G_{3} \times C_{3}^{(2)}, \mathbb{Z}\right)=C_{3}^{(3)}$, this is the same as asking whether $G_{3} \times C_{3}^{(2)}$ has a presentation of deficiency 3 . Such a presentation

$$
\begin{aligned}
\left\langle a_{1}, a_{2}, a_{3}, c\right| a_{1}^{c} & =a_{1}^{-5}, a_{2}^{c}=a_{2}^{-5},\left[a_{2}, a_{1}\right]=c^{3},\left[a_{2}^{7}, a_{1}^{7}\right]=1, \\
a_{3}^{c} & \left.=a_{3}^{2},\left[a_{3}, a_{1}\right]=a_{1}^{21},\left[a_{3}, a_{2}\right]=a_{2}^{21}\right\rangle
\end{aligned}
$$

was found for us by by M.F. Newman, so this group does not have positive relation gap after all. 
The heart of Newman's proof for the claim that (4.1) presents $G_{3} \times C_{3}^{(2)}$ is a coset enumeration showing that the group defined by (4.1) has order $7^{3} 3^{3}$. This number is also the order of $G_{3} \times C_{3}^{(2)}$, and $G_{3} \times C_{3}^{(2)}$ does have a generating set which satisfies the relations in (4.1), so nothing more is needed. The way to finding this presentation went via considering several smaller groups, and establishing (by similar methods) the following efficient presentations for the groups indicated:

$$
\begin{aligned}
& G_{2} \quad=\left\langle a_{1}, a_{2}, c \mid a_{1}^{c}=a_{1}^{2}, a_{2}^{c}=a_{2}^{2},\left[a_{2}, a_{1}\right]=c^{3}\right\rangle, \\
& G_{2} \times C_{3}=\left\langle a_{1}, a_{2}, c \mid a_{1}^{c}=a_{1}^{-5}, a_{2}^{c}=a_{2}^{2},\left[a_{2}, a_{1}\right]=c^{3}, a_{1}^{21}=1\right\rangle, \\
& G_{2} \times C_{3}^{(2)}=\left\langle a_{1}, a_{2}, c \mid a_{1}^{c}=a_{1}^{-5}, a_{2}^{c}=a_{2}^{-5},\left[a_{2}, a_{1}\right]=c^{3}, a_{1}^{21}=a_{2}^{21}=1,\left[a_{2}^{7}, a_{1}^{7}\right]=1\right\rangle
\end{aligned}
$$

and

$$
\begin{aligned}
& G_{3}=\left\langle a_{1}, a_{2}, a_{3}, c\right| a_{1}^{c}=a_{1}^{2}, a_{2}^{c}=a_{2}^{2},\left[a_{2}, a_{1}\right]=c^{3}, \\
& a_{3}^{c}\left.=a_{3}^{2},\left[a_{3}, a_{1}\right]=1,\left[a_{3}, a_{2}\right]=1\right\rangle, \\
& G_{3} \times C_{3}=\left\langle a_{1}, a_{2}, a_{3}, c\right| \begin{array}{l}
a_{1}^{c} \\
=
\end{array}=a_{1}^{-5}, a_{2}^{c}=a_{2}^{2},\left[a_{2}, a_{1}\right]=c^{3}, \\
&\left.a_{3}^{c}=a_{3}^{2},\left[a_{3}, a_{1}\right]=a_{1}^{21},\left[a_{3}, a_{2}\right]=1\right\rangle .
\end{aligned}
$$

Of course (2.6) also implies that almost all direct powers of $G_{3}$ are proficient. However, the first four are not, and the fifth is far too large a group to attempt finding an efficient presentation for it when we do not even have one for the direct cube of $G_{2}$.

\section{REFERENCES}

[1] J.L. Alperin, Local representation theory (Cambridge University Press, Cambridge, 1986).

[2] C.M. Campbell and E.F. Robertson, 'Deficiency zero presentation for $S L(2, p)$ ', Bull. London Math. Soc. 12 (1980), 17-20.

[3] C.M. Campbell, E.F. Robertson and P.D. Williams, 'Efficient presentations of direct powers of imperfect groups', Algebra Collog. 4 (1997), 21-27.

[4] J. Cossey, K.W. Gruenberg and L.G. Kovács, 'The presentation rank of a direct product of finite groups', J. Algebra 28 (1974), 597-603.

[5] C.W. Curtis and 1. Reiner, Methods of representation theory I (Wiley, New York, 1981).

[6] M.J. Dunwoody, 'Relation modules', Bull. London Math. Soc. 4 (1972), 151-155.

[7] K.W. Gruenberg, 'Über die Relationenmoduln einer endlichen Gruppe', Math. 2. 118 (1970), 30-33.

[8] Karl W. Gruenberg, Relation modules of finite groups, Conference Board of the Mathematical Sciences Regional Conference Series in Math. 25 (Amer. Math. Soc., Providence, R.I., 1976).

[9] K.W. Gruenberg, 'The partial Euler characteristics of the direct powers of a finite group', Arch. Math. 35 (1980), 267-274. 
[10] J. Harlander, 'Closing the relation gap by direct product stabilization', J. Algebra 182 (1996), 511-521.

[11] P.E. Kenne, Minimal group presentations: a computational approach, $\mathrm{PhD}$ thesis (Australian National University, Canberra, ACT, 1991).

[12] L.G. Kovács, 'Finite groups with trivial multiplicator and large deficiency', in Groups - Korea '94, (A.C. Kim and D. L. Johnson, Editors), Proc. Internat. Conf. Pusan Nat. Univ. August 18-25, 1994 (de Gruyter, Berlin, New York, 1995), pp. 211-225.

(13] R.G. Swan, 'Minimal resolutions for finite groups', Topology 4 (1965), 193-208.

[14] J.S. Wilson, Profinite groups, London Math. Soc. Monographs (Clarendon Press, Oxford, 1998).

[15] C.I. Wotherspoon, The deficiency of particular finite groups, Ph.D. Thesis (University of St. Andrews, Scotland, 1995).

Queen Mary and Westfield College

Mile End Road

London E1 4NS

England

e-mail: k.w.gruenberg@qmw.ac.uk
School of Mathematical Sciences

Australian National University

Canberra ACT 0200

Australia

e-mail: kovacs@maths.anu.edu.au 